\title{
Nonlinear signal-correction observer and application to UAV navigation
}

\author{
Xinhua Wang, Weicheng Wang
}

\begin{abstract}
A nonlinear signal-correction observer ( $\mathrm{N}$ $\mathrm{SCO}$ ) is presented for signals correction and estimation, which not only can reject the position measurement error, but also the unknown velocity can be estimated, in spite of the existence of large position measurement error and intense stochastic non-Gaussian noise. For this method, the position signal is not required to be bounded. The $\mathrm{N}$ SCO is developed for position/acceleration integration, and it is applied to an unmanned aerial vehicle (UAV) navigation: Based on the NSCO, the position and flying velocity of quadrotor UAV are estimated. An experiment is conducted to demonstrate the effectiveness of the proposed method.
\end{abstract}

Index Terms-Large position measurement error, nonlinear signal-correction observer (NSCO), UAV.

\section{INTRODUCTION}

$\mathbf{P}$ OSITION and velocity play the key roles for system navigation and control. For position recognition, global positioning system (GPS) is usually adopted and supplemented by some sensors $[1,2,3]$. On the one hand, GPS can provide the instantaneous large-error position of device, and the position signal is usually contaminated by the circumstances [2]. Velocity is also necessary for navigation and control, and the velocity of device can be estimated by the optical flow sensors (OFS) [4]. Nevertheless, they are sensible to lighting changes. The state observers $[5,6,7]$ are popular to estimate velocity from position measurement. In [5], a full-order observer was designed for the state estimation of the systems with unknown inputs. However, the above observers are not suitable to the systems with large position measurement errors. On the other hand, an inertial navigation systems (INS) with the integrators $[8,9]$ can provide position and velocity information, but it usually suffers from signal drifts over time. Several observer-based INS methods were used to estimate the position and velocity $[10,11,12]$. In [11], a dynamic observer was designed to estimate position. However, the position trajectory is required to be bounded in a constraint range, and it is not suitable to large-range navigation.

The integration of GPS and INS can limit the shortcomings of the individual systems $[13,14,15]$, and the integration can improve the accuracy of GPS outputs. The present GPS/INS

Manuscript received January 29, 2018; revised May 07, 2018 and May 31, 2018; accepted July 11, 2018. (Corresponding author: Xinhua Wang.)

Xinhua Wang is with the Department of Electrical and Electronic Engineering, University of Nottingham, Nottingham, NG7 2RD, U.K. (email: xinhua.wang1@nottingham.ac.uk; wangxinhua04@gmail.com).

Weicheng Wang is with the Huatimesee UAV Design Company, Beijing, China (e-mail: weiicheng.wang@gmail.com). integrations are mostly based on Kalman filter (KF): KF for linear systems [16], and extended Kalman filter (EKF) for the first order approximation to nonlinear systems [17]. KF (or EKF) can separate the probabilistic noise and can estimate position and velocity based on the optimization of a recursive least mean square error. However, in GPS/INS integration, the KF and EKF algorithms have several limitations: i) Noise is assumed to be zero-mean Gaussian distributed, and the process noise covariance is uncorrelated to the estimation error. As stochastic non-Gaussian noise exists in signal, the inaccurate noise information may lead to the position and velocity drifts. ii) The accurate priori information of the covariance matrices of the noises is needed. However, the noise levels may change in different applications and circumstances. iii) System linearization may cause filter divergence, and the derivation of the Jacobian matrices are nontrivial.

Considering the problems above, in this paper, a NSCO is designed to integrate the measurement signals, to reject the large position measurement error, and to estimate the unknown velocity. Inspired by the finite-time stability and robustness of autonomous systems $[18,19]$ and singular perturbations [20], the NSCO is developed, which can minimize the position sensor error, and can estimate the unknown velocity in spite of the existence of stochastic non-Gaussian noise. The parameters selection of NSCO is satisfied with the Routh-Hurwitz Stability Criterion. Moreover, the performances of the proposed NSCO are compared with those of the EKF.

As an example of application, the proposed NSCO is applied to the navigation of a quadrotor UAV, and an experiment is presented to observe the performance. In recently decades, navigation and control of aircrafts attract the investigations of many researchers $[21,22,23,24]$. Because of the great manoeuvrability and simple mechanical structure, the quadrotor UAVs can be used in exploration, security and surveillance tasks, etc. The GPS/INS navigation methods of the UAVs are usually based on KF or EKF with some supplemented sensors [25], and the noise is assumed to be be zero-mean Gaussian distributed. Some navigation tools of the UAVs adopt vision systems [26,27]. The complex environments or adverse light effects can make vision systems difficult to recognize the position and velocity. In this paper, the following scenario of quadrotor UAV navigation is considered: large measurement error exists in position signal; stochastic non-Gaussian noise exists in the acceleration and position; the flying velocity is unknown. In this UAV navigation system, the proposed NSCO is adopted to estimate the position and velocity. Finally, two simple controllers based on the NSCO are designed to stabilize 
the flight dynamics.

\section{CONFIGURATION OF NSCO}

The goal of the NSCO design is to reject the position measurement error, and to estimate the unknown velocity through the signals integration.

\section{A. Case of position/acceleration integration}

We consider the following case of system navigation:

1) GPS provides the large-error position $a_{1}(t)=a_{01}(t)+$ $d_{1}(t)$, where $a_{01}(t)$ is real position, $d_{1}(t)$ is the large measurement error, and $\sup _{t \in[0, \infty)}\left|d_{1}(t)\right| \leq L_{1}<\infty$;

2) INS gives the acceleration $a_{3}(t)=a_{03}(t)+d_{3}(t)$, where $a_{03}(t)$ is real acceleration, $d_{3}(t)$ is the measurement error, $\sup _{t \in[0, \infty)}\left|d_{3}(t)\right| \leq L_{3}<\infty$, and $L_{3} \ll L_{1}$;

3) The velocity $a_{02}(t)$ is unknown.

For the real position $a_{01}(t)$, velocity $a_{02}(t)$ and acceleration $a_{03}(t)$, the relations $a_{01}(t)=\int_{0}^{t} \int_{0}^{v} a_{03}\left(\sigma_{1}\right) d \sigma_{1} d \sigma_{2}$ and $a_{02}(t)=\int_{0}^{t} a_{03}(\sigma) d \sigma$ hold.

Question: How to estimate the real position $a_{01}(t)$ and the velocity $a_{02}(t)$ of device from the measurements of the position $a_{1}(t)=a_{01}(t)+d_{1}(t)$ and the acceleration $a_{3}(t)=$ $a_{03}(t)+d_{3}(t)$, in spite of the existence of the large position measurement error and stochastic non-Gaussian noise?

\section{B. Definition of NSCO}

The usual observers, for instance, sliding mode observers, can estimate the unknown states based on the small-error position measurement $a_{1}(t)$. For the following sliding mode observer (See Theorem 6 in [28])

$$
\begin{aligned}
& \dot{x}_{1}=x_{2}-\lambda_{1}\left|x_{1}-a_{1}(t)\right|^{\frac{2}{3}} \operatorname{sign}\left(x_{1}-a_{1}(t)\right) \\
& \dot{x}_{2}=x_{3}-\lambda_{2}\left|x_{1}-a_{1}(t)\right|^{\frac{1}{3}} \operatorname{sign}\left(x_{1}-a_{1}(t)\right) \\
& \dot{x}_{3}=-\lambda_{3} \operatorname{sign}\left(x_{1}-a_{1}(t)\right)
\end{aligned}
$$

if the sensor error exists in the measurement signal $a_{1}(t)$, i.e., $a_{1}(t)=a_{01}(t)+d_{1}(t)$, where $a_{01}(t)$ is real position, $d_{1}(t)$ is the small position measurement error, $\sup _{t \in[0, \infty)}\left|d_{1}(t)\right| \leq$ $L_{1}<\infty$, then there exist $\mu>0$ and $t_{s}>0$, such that, for $t \geq t_{s}$

$$
\left|x_{i}-a_{0 i}(t)\right| \leq \mu L_{1}^{\frac{4-i}{3}}, i=1,2,3
$$

The observer is suitable to estimate velocity $a_{02}(t)$ and acceleration $a_{03}(t)$ from the small-error position measurement $a_{1}(t)=a_{01}(t)+d_{1}(t)$. However, they cannot reject sufficiently the effect of large position error $d_{1}(t)$ : if the large error $d_{1}(t)$ exists in the measurement signal $a_{1}(t)=a_{01}(t)+d_{1}(t)$, the up-boundness $L_{1}^{\frac{4-i}{3}}$ of the estimation errors is also large. Therefore, the usual observers are not suitable to the systems with large position measurement errors.

Actually, the measurements for the usual navigation systems include the large-error position measurement by GPS and the small-error acceleration measurement by INS. For system navigation, the accurate position and velocity are needed.
Definition 1 (NSCO): For the large-error position measurement $a_{1}(t)=a_{01}(t)+d_{1}(t)$ and small-error acceleration measurement $a_{3}(t)=a_{03}(t)+d_{3}(t)$, where, $d_{1}(t)$ and $d_{3}(t)$ are the unknown large and small measurement errors, respectively, a nonlinear signal-correction observer (NSCO) is designed, such that the large position measurement error can be rejected and the unknown velocity is estimated.

The configuration of a NSCO is described by

$$
\begin{aligned}
& \dot{x}_{1}=x_{2} ; \quad \dot{x}_{2}=x_{3} ; \\
& \dot{x}_{3}=f\left(x_{1}-a_{1}(t), x_{2}, x_{3}-a_{3}(t)\right)
\end{aligned}
$$

with the conclusions:

$$
x_{1} \rightarrow a_{01}(t), x_{2} \rightarrow a_{02}(t), x_{3} \rightarrow a_{03}(t)
$$

In the above NSCO configuration, the system inputs include the position measurement $a_{1}(t)$ and acceleration measurement $a_{3}(t)$. The states $x_{1}, x_{2}$ and $x_{3}$ estimate the real position $a_{01}(t)$, velocity $a_{02}(t)$ and acceleration $a_{03}(t)$, respectively. Importantly, the large sensor error $d_{1}(t)$ in position measurement $a_{1}(t)$ will be rejected sufficiently, and the position and velocity drifts will be avoided even non-Gaussian noise exists.

\section{DESIGN OF NSCO}

In the following, considering the large position sensor error and stochastic non-Gaussian noise, a NSCO is designed to to reject the position measurement error and to estimate the velocity. The position is not required to be bounded. One theorem is presented as follows.

\section{A. NSCO for position/acceleration integration}

Theorem 1: Considering Case A in Section II, for system

$$
\begin{aligned}
\dot{x}_{1}= & x_{2} ; \quad \dot{x}_{2}=x_{3} ; \\
\varepsilon^{4} \dot{x}_{3}= & -k_{1}\left|\varepsilon\left(x_{1}-a_{1}(t)\right)\right|^{\alpha_{1}} \operatorname{sign}\left(x_{1}-a_{1}(t)\right) \\
& -k_{2}\left|\varepsilon^{2} x_{2}\right|^{\alpha_{2}} \operatorname{sign}\left(x_{2}\right) \\
& -k_{3}\left|\left(x_{3}-a_{3}(t)\right)\right|^{\alpha_{3}} \operatorname{sign}\left(x_{3}-a_{3}(t)\right)
\end{aligned}
$$

where $\varepsilon \in(0,1)$ is the perturbation parameter;

$$
k_{1}>0, k_{3}>0, k_{2}>\varepsilon^{3 \alpha_{3}} \frac{k_{1}}{k_{3}}
$$

and $\alpha_{1}, \alpha_{2}, \alpha_{3}$ satisfy

$$
\alpha_{3} \in(0,1), \alpha_{2}=\frac{\alpha_{3}}{2-\alpha_{3}}, \alpha_{1}=\frac{\alpha_{3}}{3-2 \alpha_{3}}
$$

1) if the sensor errors exist in the measurement signals $a_{1}(t)$ and $a_{3}(t)$, i.e., $a_{1}(t)=a_{01}(t)+d_{1}(t)$ and $a_{3}(t)=a_{03}(t)+$ $d_{3}(t)$, where $a_{01}(t)$ is real position, $d_{1}(t)$ is the large position measurement error, $\sup _{t \in[0, \infty)}\left|d_{1}(t)\right| \leq L_{1}<\infty, a_{03}(t)$ is real acceleration, $d_{3}(t)$ is the acceleration measurement error, $\sup _{t \in[0, \infty)}\left|d_{3}(t)\right| \leq L_{3}<\infty$, and $L_{3} \ll L_{1}$, then there exist $L>0, \gamma>\frac{4}{\alpha_{1}}$ and $\Gamma>0$, such that, for $t \geq \varepsilon \Gamma(\Xi(\varepsilon) e(0))$,

$$
\left|x_{i}-a_{0 i}(t)\right| \leq L\left(\delta_{d i}\right)^{\gamma}, i=1,2,3
$$


where, $\delta_{d i}=\varepsilon^{\alpha_{1}-\frac{i}{\gamma}}+\frac{2^{1-\alpha_{3}} k_{3} L_{3}^{\alpha_{3}}}{\delta_{0}} \varepsilon^{-\frac{i}{\gamma}} \in(0,1), i=1,2,3$; $\delta_{0}=\sum_{i=1}^{2} 2^{1-\alpha_{i}} k_{i} L_{i}^{\alpha_{i}}+L_{a} ; \sup _{t \in[0, \infty)}\left|\dot{a}_{03}(t)\right| \leq L_{a}<\infty$; $\gamma=(1-\beta) / \beta, \beta \in\left(0, \alpha_{1} /\left(\alpha_{1}+4\right)\right) ; \Xi(\varepsilon)=\operatorname{diag}\left\{\varepsilon, \varepsilon^{2}, \varepsilon^{3}\right\}$, and $e_{i}=x_{i}-a_{0 i}(t), i=1,2,3 ; e=\left[\begin{array}{lll}e_{1} & e_{2} & e_{3}\end{array}\right]^{T}$.

2) Furthermore, assume there is no sensor error in signal $a_{3}(t)$, i.e., $a_{3}(t)=a_{03}(t)$, then, even large sensor error exists in signal $a_{1}(t)$, for $t \geq \varepsilon \Gamma(\Xi(\varepsilon) e(0))$,

$$
\left|x_{i}-a_{0 i}(t)\right| \leq L \varepsilon^{\alpha_{1} \gamma-i}, i=1,2,3
$$

The proof of Theorem 1 is presented in Appendix.

\section{B. Robustness analysis in frequency domain}

In practice, high-frequency noises exist in measurements $a_{1}(t)$ and $a_{3}(t)$. For the NSCO, frequency-sweep method [29] can be used to approximately analyse the nonlinear behaviors of the NSCO, and the Bode plots are adopted to describe the system frequency characteristics. By frequency-sweep method, we can find that the NSCO leads to perform precise estimation and strong rejection of high-frequency noise.

The test of frequency characteristic can be implemented by Bode plot fitting. The input signals are selected as: acceleration $a_{3}(t)=A_{m} \sin (\omega t)$, and position $a_{1}(t)=-\frac{A_{m}}{\omega^{2}} \sin (\omega t)$, where $A_{m}$ and $\omega$ are the amplitude and angular frequency of the input signal $a_{3}(t)$, respectively. The outputs are $x_{3}, x_{2}$ and $x_{1}$. Equivalently, $a_{3}(t)$ can be taken as the unique input signal, and $a_{1}(t)$ is the double integral of $a_{3}(t)$. The Bode plots of the relations $a_{3}(t) \rightarrow x_{3}, a_{3}(t) \rightarrow x_{2}$ and $a_{3}(t) \rightarrow x_{1}$ will be sketched, respectively. In fact, for the above input-output relations, the ideal operators are $1,1 / s$ and $1 / s^{2}$, respectively.

For the NSCO, the parameters are selected as follows: $k_{1}=0.5, k_{2}=0.2, k_{3}=10 ; \alpha_{3}=\alpha=0.8,0.5,0.3$; $\varepsilon=0.6,0.4,0.25$, respectively. The Bode plots with different selections of $\varepsilon$ and $\alpha_{3}$ are described in Fig.1: Figs.1(a)(c) present the frequency characteristics of the acceleration, velocity and position estimations, respectively.

Comparing with ideal operators $1 / s$ and $1 / s^{2}$, not only the NSCO can obtain their estimations of velocity and position precisely, but also the high-frequency noise is rejected sufficiently: as $\omega \rightarrow \infty$, the magnitude tends $-\infty$.

Parameter $\varepsilon$ affects the low-pass frequency bandwidth: Decreasing the perturbation parameter $\varepsilon$, the low-pass frequency bandwidth becomes larger, and the estimation speed becomes fast; on the other hand, increasing perturbation parameter $\varepsilon$, the low-pass frequency bandwidth becomes smaller, and much noise can be rejected sufficiently (See the cases of $\varepsilon=0.6$, $\varepsilon=0.4$ and $\varepsilon=0.25$ in Figs.1 (a)-(c), respectively). Parameter $\alpha_{3} \in(0,1)$ affects the decay speed of frequency characteristic curves near the cut-off frequency (See the cases of $\alpha_{3}=\alpha=0.8,0.5,0.3$ in Fig.1, respectively): smaller $\alpha_{3} \in(0,1)$ can obtain more precise estimations; on the other hand, larger $\alpha_{3} \in(0,1)$ can reduce much noise, however, a bit estimation delay happens.

Remark 1 (Analysis of NSCO):

1) Stability and robustness of NSCO: In NSCO (5), $x_{i}$ estimates the desired values $a_{0 i}(t), i=1,2,3$, respectively. In

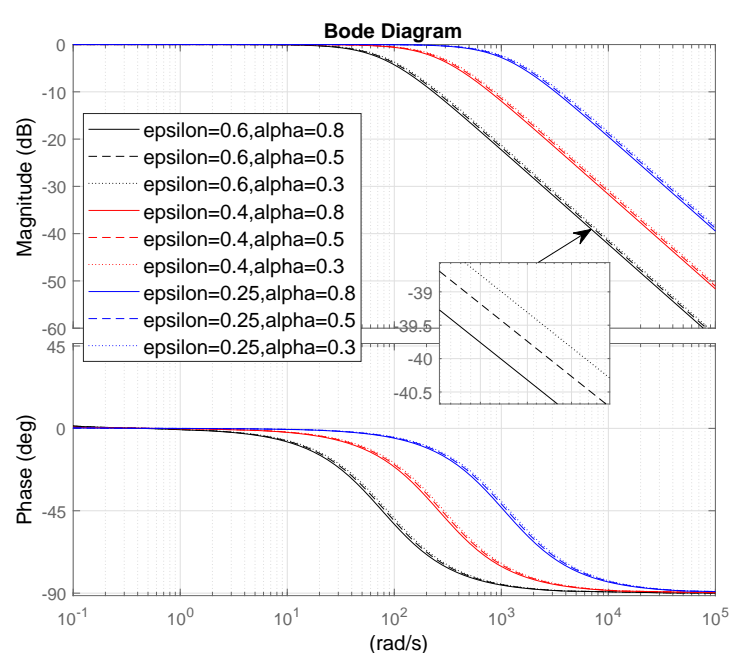

(a)

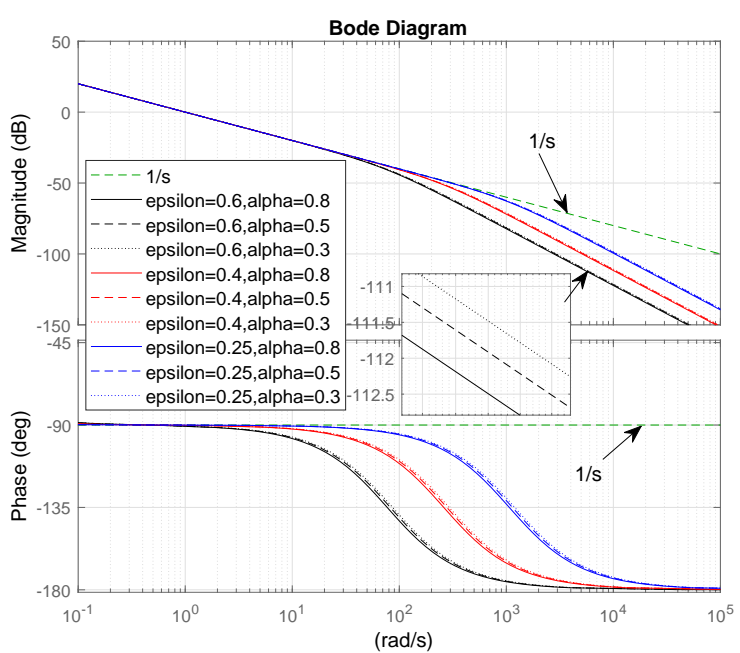

(b)

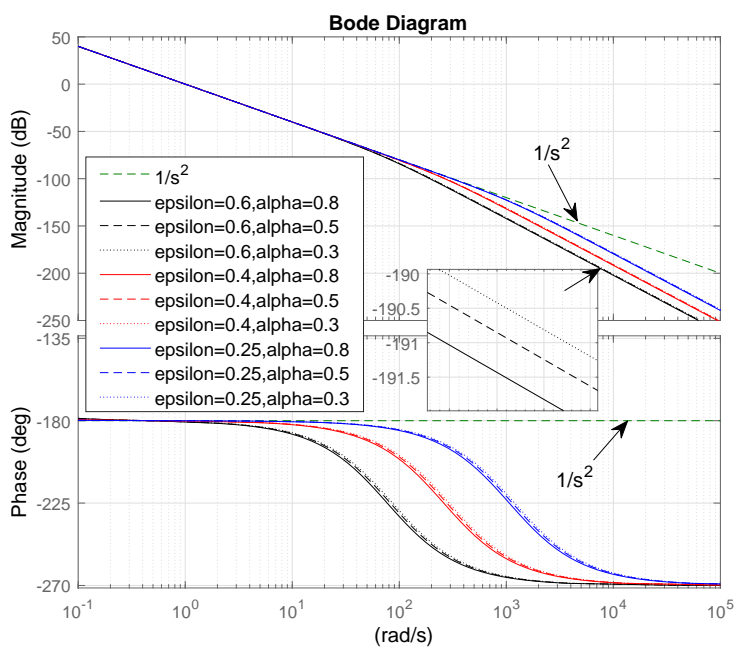

(c)

Fig. 1. Frequency characteristics with changes of $\varepsilon$ and $\alpha$ for NSCO. (a) Acceleration estimate. (b) Velocity estimate. (c) Position estimate. 
the estimation error (9), due to $\varepsilon \in(0,1)$ and $\alpha_{1} \gamma-i \gg 1$, the up-boundness of estimation error $L \varepsilon^{\alpha_{1} \gamma-i}$ (where, $i=1,2,3$ ) is sufficiently small. Therefore, the NSCO leads to perform strong rejection of persistent sensor errors and disturbances, and high-precision signal estimations are achieved. Furthermore, from the frequency-domain analysis (See Fig. 1), the NSCO can reject the high-frequency noise.

2) Large position error rejection and velocity estimation: In the NSCO (5), the position is not required to be bounded. The large sensor error $d_{1}(t)$ (where, $\sup _{t \in[0, \infty)}\left|d_{1}(t)\right| \leq L_{1}<\infty$ ) in the position measurement $a_{1}(t)=a_{01}(t)+d_{1}(t)$ can be rejected sufficiently. In fact, based on the singular perturbation technique and nonlinear contraction mapping theory, from (30) in the proof of Theorem 1, the effect of the sensor error $d_{1}(t)$ is compressed into $2^{1-\alpha_{1}} k_{1} L_{1}^{\alpha_{1}} \varepsilon^{\alpha_{1}}$. Furthermore, in the estimation error (9), due to $\varepsilon \in(0,1)$, the up-boundness of the position estimate error $L \varepsilon^{\alpha_{1} \gamma-1}$ is sufficiently small, and $L \varepsilon^{\alpha_{1} \gamma-1} \ll L_{1}$. Therefore, the measurement error in position signal can be rejected sufficiently.

In the estimate error (9), due to $\varepsilon \in(0,1)$, the up-boundness of the velocity estimate error $L \varepsilon^{\alpha_{1} \gamma-2}$ is small enough.

3) No drift phenomenon: From (8), in spite of the existence of the large sensor error and non-Gaussian noise, the estimate errors are bounded, and their up-boundnesses are unrelated to time after $t \geq \varepsilon \Gamma(\Xi(\varepsilon) e(0))$. Therefore, even for unbounded position navigation, no drift phenomenon happens.

Remark 2 (The rules of NSCO parameters selection):

For the NSCO, there are several rules on the parameters selection:

1) Basic stability condition: The parameters $\left(k_{1}, k_{2}, k_{3}\right)$ and $\left(\alpha_{1}, \alpha_{2}, \alpha_{3}\right)$ are satisfied with Eqs. (6) and (7), respectively.

2) For rejecting the measurement error in position: When the measurement error $d_{1}(t)$ in position signal $a_{1}(t)$ increases, i.e., $L_{1}$ increases, in order to decrease the error effect $k_{1} L_{1}^{\alpha_{1}}$ of $\delta_{0}=\sum_{i=1}^{2} 2^{1-\alpha_{i}} k_{i} L_{i}^{\alpha_{i}}+L_{a}$ in (30), parameter $k_{1}>0$ decreases to improve the estimate precisions. Furthermore, in order to decrease $L_{1}^{\alpha_{1}}, \alpha_{1} \in(0,1)$ should decrease to improve the estimate precisions.

3) For low-pass filtering: In order to increase the estimation speed, $\varepsilon \in(0,1)$ should decrease to make the low-pass frequency bandwidth larger, or $\alpha_{3} \in(0,1)$ decreases. If much noise exists, $\varepsilon$ should increase, or $\alpha_{3} \in(0,1)$ increases. Thus, the low-pass frequency bandwidth becomes smaller, and the noise can be rejected sufficiently (See Fig. 1).

\section{UAV NAVIGATION BASED ON NSCO}

A quadrotor UAV navigation is studied. In this scenario, the large-error position measurement of UAV is considered. The forces and torques of quadrotor UAV are described in Fig. 2. The UAV is controlled by the thrust forces $F_{i}(i=1,2,3,4)$ which are generated by four propellers.

\section{A. Quadrotor UAV dynamics}

Let $\Xi_{g}=\left(E_{x}, E_{y}, E_{z}\right)$ and $\Xi_{b}=\left(E_{x}^{b}, E_{y}^{b}, E_{z}^{b}\right)$ denote the inertial and fuselage frames, respectively; $\psi, \theta$ and $\phi$ are the Euler angles expressed in the yaw, pitch and roll angles,

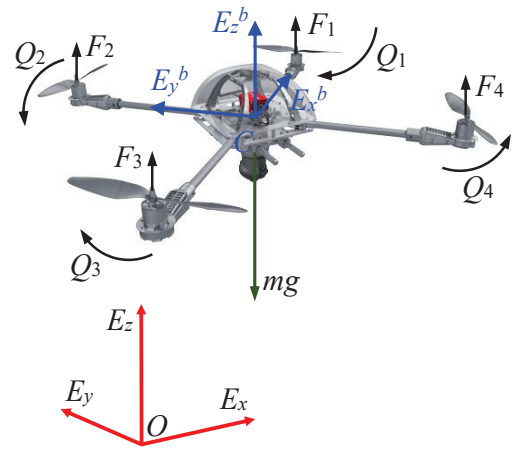

Fig. 2. Forces and torques of quadrotor UAV.

respectively; The symbols $c_{\theta}$ and $s_{\theta}$ are used for $\cos \theta$ and $\sin \theta$, respectively. $F_{i}=b \omega_{i}^{2}$ is the thrust force by rotor $i$, and the reactive torque generated by rotor $i$ is written as $Q_{i}=k \omega_{i}^{2}$. Therefore, $Q_{i}=\frac{k}{b} F_{i}$ holds. The total thrust by the four rotors is given by $F=\sum_{i=1}^{4} F_{i}$. The motion equations of the UAV in the coordinate $(x, y, z)$ are then

$$
\begin{gathered}
m \ddot{x}=\left(c_{\psi} s_{\theta} c_{\phi}+s_{\psi} s_{\phi}\right) F-k_{x} \dot{x}+\Delta_{x} \\
m \ddot{y}=\left(s_{\psi} s_{\theta} c_{\phi}-c_{\psi} s_{\phi}\right) F-k_{y} \dot{y}+\Delta_{y} \\
m \ddot{z}=c_{\theta} c_{\phi} F-m g-k_{z} \dot{z}+\Delta_{z} \\
J_{\psi} \ddot{\psi}=u_{\psi}-k_{\psi} \dot{\psi}+\Delta_{\psi} \\
J_{\theta} \ddot{\theta}=u_{\theta}-l k_{\theta} \dot{\theta}+\Delta_{\theta} \\
J_{\phi} \ddot{\phi}=u_{\phi}-l k_{\phi} \dot{\phi}+\Delta_{\phi}
\end{gathered}
$$

where, $k_{x}, k_{y}, k_{z}, k_{\psi}, k_{\theta}$ and $k_{\phi}$ are the drag coefficients; $\left(\Delta_{x}, \Delta_{y}, \Delta_{z}\right)$ and $\left(\Delta_{\psi}, \Delta_{\theta}, \Delta_{\phi}\right)$ are the bounded uncertainties in position and attitude dynamics, respectively; $J=$ $\operatorname{diag}\left\{J_{\psi}, J_{\theta}, J_{\phi}\right\}$ is the matrix of the three-axis moment of inertias; and

$$
\begin{aligned}
& u_{\psi}=\frac{k}{b} \sum_{i=1}^{4}(-1)^{i+1} F_{i}, u_{\theta}=\left(F_{3}-F_{1}\right) l \\
& u_{\phi}=\left(F_{2}-F_{4}\right) l
\end{aligned}
$$

The attitude information $(\psi, \theta, \phi, \dot{\psi}, \dot{\theta}, \dot{\phi})$ is measured by an IMU. The triaxial acceleration vector $\ddot{X}_{b}=\left[\begin{array}{lll}\ddot{x}_{b} & \ddot{y}_{b} & \ddot{z}_{b}\end{array}\right]^{T}$ in body frame $\Xi_{b}$ is obtained by the triaxial accelerometer in the IMU. Therefore, the acceleration vector $\ddot{X}=$ $\left[\begin{array}{lll}\ddot{x} & \ddot{y} & \ddot{z}\end{array}\right]^{T}$ in inertial frame $\Xi_{g}$ can be written as $\ddot{X}=$ $R_{b g} \ddot{X}_{b}+\left[\begin{array}{ccc}0 & 0 & g\end{array}\right]^{T}$.

For the UAV, we are interested in using the NSCO to estimate $(x, y, z, \dot{x}, \dot{y}, \dot{z})$ from the acceleration and largeerror position measurements.

\section{B. Controller design}

In this section, the control laws are designed for trajectory tracking and attitude stabilization. For the reference trajectory 
$\left(x_{d}, y_{d}, z_{d}\right)$, the error system of position dynamics (10) can be given by

$$
\ddot{e}_{p}=m^{-1}\left(u_{p}+\Xi_{p}+\delta_{p}\right)
$$

where, $e_{1}=x-x_{d}, e_{2}=\dot{x}-\dot{x}_{d}, e_{3}=y-y_{d}, e_{4}=\dot{y}-\dot{y}_{d}$, $e_{5}=z-z_{d}, e_{6}=\dot{z}-\dot{z}_{d} ;$ and

$$
\begin{aligned}
& e_{p}=\left[\begin{array}{l}
e_{1} \\
e_{3} \\
e_{5}
\end{array}\right], u_{p}=\left[\begin{array}{c}
c_{\psi} s_{\theta} c_{\phi}+s_{\psi} s_{\phi} \\
s_{\psi} s_{\theta} c_{\phi}-c_{\psi} s_{\phi} \\
c_{\theta} c_{\phi}
\end{array}\right] F, \\
& \Xi_{p}=\left[\begin{array}{c}
-m \ddot{x}_{d} \\
-m \ddot{y}_{d} \\
-m \ddot{z}_{d}-m g
\end{array}\right], \delta_{p}=\left[\begin{array}{c}
\Delta_{x}-k_{x} \dot{x} \\
\Delta_{y}-k_{y} \dot{y} \\
\Delta_{z}-k_{z} \dot{z}
\end{array}\right]
\end{aligned}
$$

For the reference attitude $\left(\psi_{d}, \theta_{d}, \phi_{d}\right)$, the error system of attitude dynamics (11) is written as

$$
\ddot{e}_{a}=J^{-1}\left(u_{a}+\Xi_{a}+\delta_{a}\right)
$$

where, $e_{7}=\psi-\psi_{d}, e_{8}=\dot{\psi}-\dot{\psi}_{d}, e_{9}=\theta-\theta_{d}, e_{10}=\dot{\theta}-\dot{\theta}_{d}$, $e_{11}=\phi-\phi_{d}, e_{12}=\dot{\phi}-\dot{\phi}_{d}$;

$$
\begin{gathered}
e_{a}=\left[\begin{array}{c}
e_{7} \\
e_{9} \\
e_{11}
\end{array}\right], \Xi_{a}=\left[\begin{array}{c}
-J_{\psi} \ddot{\psi}_{d} \\
-J_{\theta} \ddot{\theta}_{d} \\
-J_{\phi} \ddot{\phi}_{d}
\end{array}\right], \\
u_{a}=\left[\begin{array}{c}
u_{\psi} \\
u_{\theta} \\
u_{\phi}
\end{array}\right], \delta_{a}=\left[\begin{array}{c}
\Delta_{\psi}-k_{\psi} \dot{\psi} \\
\Delta_{\theta}-l k_{\theta} \dot{\theta} \\
\Delta_{\phi}-l k_{\phi} \dot{\phi}
\end{array}\right]
\end{gathered}
$$

1) Position dynamics controller: Based on the NSCO (5), for position dynamics (10), to track reference trajectory $\left(x_{d}, y_{d}, z_{d}\right)$, a controller is selected as

$$
u_{p}=-\Xi_{p}-\widehat{\delta}_{p}-m\left(k_{p 1} \widehat{e}_{p}+k_{p 2} \widehat{\dot{e}}_{p}\right)
$$

where, $k_{p 1}, k_{p 2}>0$. Therefore, position error system (13) by controller (17) converges to the origin asymptotically, i.e., $e_{p} \rightarrow 0$ and $\dot{e}_{p} \rightarrow 0$ as $t \rightarrow \infty$, where the variables $\widehat{e}_{1}=$ $\widehat{x}-x_{d}, \widehat{e}_{2}=\widehat{\dot{x}}-\dot{x}_{d}, \widehat{e}_{3}=\widehat{y}-y_{d}, \widehat{e}_{4}=\widehat{\dot{y}}-\dot{y}_{d}, \widehat{e}_{5}=\widehat{z}-z_{d}$ and $\widehat{e}_{6}=\widehat{\dot{z}}-\dot{z}_{d}$ are estimated by $\operatorname{NSCO}(5)$; and

$$
\widehat{e}_{p}=\left[\begin{array}{c}
\widehat{e}_{1} \\
\widehat{e}_{3} \\
\widehat{e}_{5}
\end{array}\right], \widehat{\dot{e}}_{p}=\left[\begin{array}{c}
\widehat{e}_{2} \\
\widehat{e}_{4} \\
\widehat{e}_{6}
\end{array}\right], \widehat{\delta}_{p}=m \ddot{X}-u_{p}
$$

From (14) and (17), we deduce the total thrust

$$
F=\left\|-\Xi_{p}-\widehat{\delta}_{p}-m\left(k_{p 1} \widehat{e}_{p}+k_{p 2} \widehat{\dot{e}}_{p}\right)\right\|_{2}
$$

2) Attitude dynamics controller: Firstly, a small change is operated for the continuous differentiator in [30] to become the following extended observer, and to estimate the uncertainty $\delta_{a}=\left[\begin{array}{ccc}\Delta_{\psi} & \Delta_{\theta} & \Delta_{\phi}\end{array}\right]^{T}$ in the attitude dynamics (11):

$$
\begin{aligned}
& \dot{x}_{1 *}=x_{2 *}-\lambda_{1 *}\left|x_{1 *}-\omega_{*}\right|^{\frac{1+\alpha_{*}}{2}} \operatorname{sign}\left(x_{1 *}-\omega_{*}\right)+\Omega_{*} \\
& \dot{x}_{2 *}=-\lambda_{2 *}\left|x_{1 *}-\omega_{*}\right|^{\alpha_{*}} \operatorname{sign}\left(x_{1 *}-\omega_{*}\right)
\end{aligned}
$$

with $*=\{\psi, \theta, \phi\}$, then, from Theorem 1 in [30], there exist a finite time $t_{s}>0$ such that, for $t \geq t_{s}$,

$$
x_{1 *}=\omega_{*}, x_{2 *}=\Delta_{*} / J_{*}
$$

where $\lambda_{1 *}, \lambda_{2 *}>0, \alpha_{*} \in(0,1), \omega_{*}$ is the angular velocity measurement, and

$$
\begin{aligned}
\Omega_{\psi} & =\frac{k}{b J_{z}} \sum_{i=1}^{4}(-1)^{i+1} F_{i}-k_{\psi} \dot{\psi} / J_{z} \\
\Omega_{\theta} & =\left(F_{3}-F_{1}\right) l / J_{y}-l k_{\theta} \dot{\theta} / J_{y} \\
\Omega_{\phi} & =\left(F_{2}-F_{4}\right) l / J_{x}-l k_{\phi} \dot{\phi} / J_{x}
\end{aligned}
$$

From (21) and (16), we obtain

$$
\widehat{\delta}_{a}=\left[\begin{array}{lll}
J_{\psi} x_{2 \psi} & J_{\theta} x_{2 \theta} & J_{\phi} x_{2 \phi}
\end{array}\right]^{T}
$$

The continuous observer can provide continuous, accurate and smooth estimations, reducing high frequency vibrations and improving overall control performance.

For attitude dynamics (11), to track reference attitude $\left(\psi_{d}, \theta_{d}, \phi_{d}\right)$, a controller can be selected as

$$
u_{a}=-\Xi_{a}-\widehat{\delta}_{a}-J\left(k_{a 1} e_{a}+k_{a 2} \dot{e}_{a}\right)
$$

where, $k_{a 1}, k_{a 2}>0$, then attitude error system (15) by controller (24) converges to the origin asymptotically, i.e., $e_{a} \rightarrow 0$ and $\dot{e}_{a} \rightarrow 0$ as $t \rightarrow \infty$.

\section{EXPERIMENT ON UAV NAVIGATION}

In this section, the experimental results are given to illustrate the performance of the proposed scheme. The platform of quadrotor UAV navigation and control is shown in Fig. 3, and the UAV parameters are given in Table I. The flight control system implementation on the hardware is shown in Fig.4. The implementation of the navigation strategy based on NSCO is done in the platform setup, whose components are: Arduino Mega 2560 (sampling frequency: $16 \mathrm{MHz}) \rightarrow$ (CPU clock rate (or speed)): 16MHz. Gumstix microcomputer and an Arduino Mega 2560 are taken as the driven boards, which have multiple PWM output channels. An IMU (XsensMTI AHRS) is used to measure the attitude, whose sampling frequency is $10 \mathrm{kHz}$. Also, the triaxial acceleration vector in body frame is obtained by the triaxial accelerometer in the IMU. The control update time is $5 \mathrm{~ms}$.

Real position for comparison: The Vicon system (i.e., indoor motion capture system) is an indoor positioning system with a sub-millimeter precision. Therefore, the position from the Vicon system can be taken as the real position of UAV, and it will be compared with the estimation from the NSCO based on the large-error position measurement.

Large-error position measurement: Because the GPS signal cannot be obtained in the room of lab, we use the output of the Vicon system plus the prior GPS error signal as the largeerror position measurement (See Fig. 4). Here, the GPS error signal was obtained through an outdoor GPS positioning test: Using a GPS receiver, the position signal of a static object at the origin was recorded in 100 seconds. In order to get the 

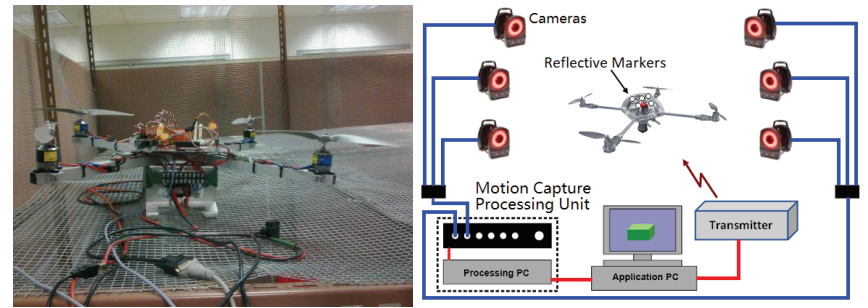

Fig. 3. Platform of quadrotor UAV system.

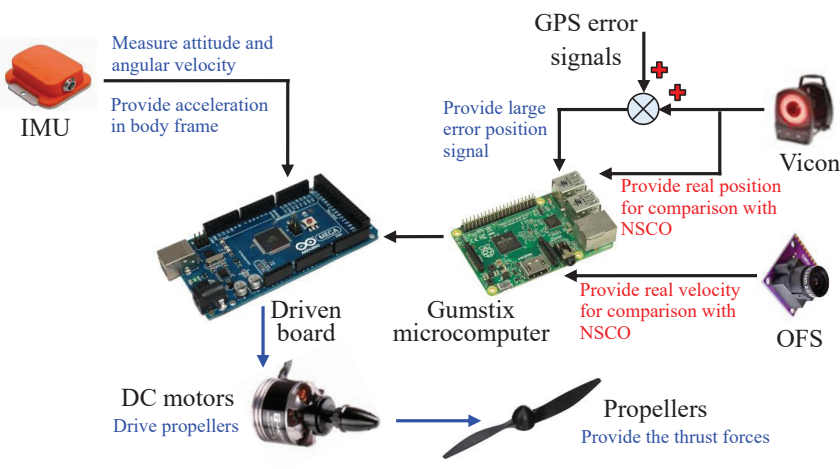

Fig. 4. Flight control system implementation on the hardware.

TABLE I

UAV PARAMETERS

\begin{tabular}{lll}
\hline \hline Symbol & \multicolumn{1}{c}{ Quantity } & \multicolumn{1}{c}{ Value } \\
\hline$m$ & mass of UAV & $2.01 \mathrm{~kg}$ \\
$g$ & gravity & $9.81 \mathrm{~m} / \mathrm{s}^{2}$ \\
$l$ & distance between rotor and gravity center & $0.2 \mathrm{~m}$ \\
$J_{\phi}$ & moment of inertia about roll & $1.25 \mathrm{~kg} \cdot \mathrm{m}^{2}$ \\
$J_{\theta}$ & moment of inertia about pitch & $1.25 \mathrm{~kg} \cdot \mathrm{m}^{2}$ \\
$J_{\psi}$ & moment of inertia about yaw & $2.5 \mathrm{~kg} \cdot \mathrm{m}^{2}$ \\
$b$ & rotor force coefficient & $2.923 \times 10^{-3}$ \\
$k$ & Rotor torque coefficient & $5 \times 10^{-4}$ \\
\hline
\end{tabular}

much larger measurement error, the recorded signal magnified 3 times. Then, we obtained the GPS error signal.

Real velocity for comparison: A XZN Optical flow sensor (OFS) board (up to $6400 \mathrm{fps}$ update rate, 30x30 pixel resolution) is used to measure the velocity, and the value is compared with the estimation from the NSCO. It is noted that this OFS is more suitable to use indoor because it is sensible to lighting changes. Therefore, we can regulate the indoor light to obtain the ideal OFS measurement.

Reference trajectory: Tracking desired position trajectory is studied. The desired trajectory consists of takeoff and a circle with the radius $5 \mathrm{~m}$, velocity $1 \mathrm{~m} / \mathrm{s}$ and altitude $3 \mathrm{~m}$, which is shown in Fig. 5.

The NSCO (5) estimates the position and velocity from the contaminated position and acceleration measurements. Controllers (17) and (24) are adopted to drive the UAV to track the reference trajectory. The parameters of NSCOs are: $\alpha_{i, 3}=0.5, k_{i, 1}=0.5, k_{i, 2}=0.2, k_{i, 3}=10,1 / \varepsilon_{i}=4$, $i=1,2,3$. The controller gains are: $k_{p 1}=3.2, k_{p 2}=5$,

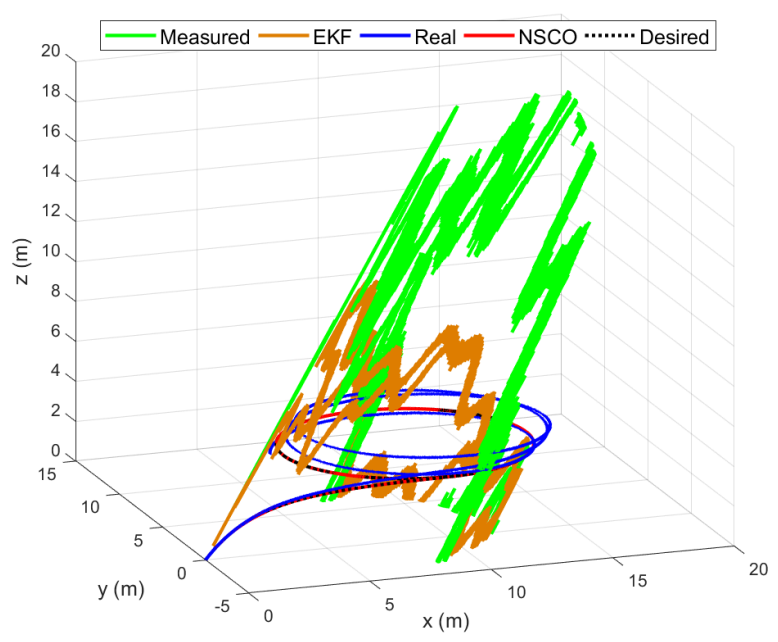

(a)

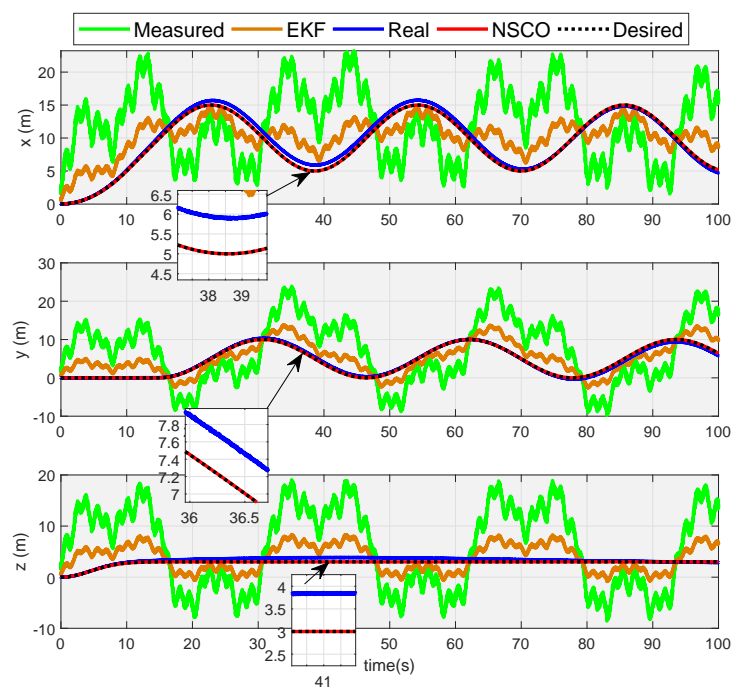

(b)
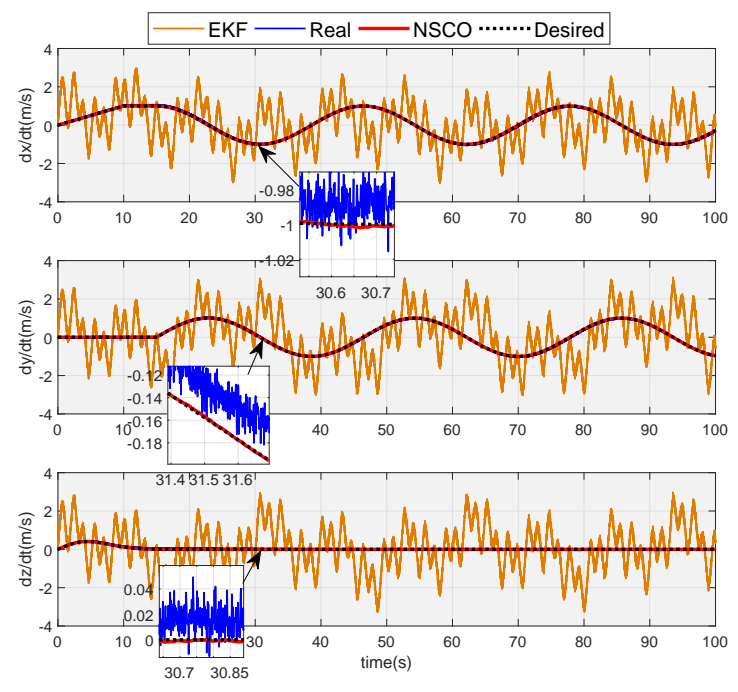

(c)

Fig. 5. UAV navigation based on NSCO. (a) Navigation trajectories. (b) Position estimate. (c) Velocity estimate. 
$k_{a 1}=2.6, k_{a 2}=3.5$.

The NSCO provides the estimations of the position and velocity, which are replaced into the controller. Now, the $\mathrm{N}$ SCO performance is studied through the behavior of estimated position and velocity, and compared with the estimations by the EKF given in [17]. The estimations of the states by the EKF consider the same conditions.

Fig. 5(a) displays the estimated trajectories by the NSCO and EKF. In addition, the estimation comparisons of the threedirection positions are shown in Fig. 5(b): The sensor errors of position measurements are $20 \mathrm{~m}$. The errors by the NSCO are less than $1 \mathrm{~m}$, while the up-boundness of the estimate errors by the EKF is $6 \mathrm{~m}$ for all the positions. The large measurement errors and stochastic noises are rejected sufficiently by the NSCO. Importantly, even in the long-time flight (1000s), no drift phenomenon happened. However, comparing to the $\mathrm{NSCO}$, the larger position estimation errors exist by the EKF, and the EKF can't restrain efficiently the effect of stochastic non-Gaussian noise. Fig. 5(c) illustrates the comparison of velocity estimations between the NSCO and the EKF, where the velocity estimations from the NSCO showed the smaller error estimations.

Importantly, we found that the rules of NSCO parameters selection (Remark 2) are confirmed by tuning parameters of NSCO in the experiment: 1$)$ For the parameter $\varepsilon \in(0,1)$ : on the one hand, if $\varepsilon$ decreases, the estimation speed will increase, and the low-pass frequency bandwidth will become larger; on the other hand, $\varepsilon$ increases, much noise will be rejected. 2) The smaller $k_{1}>0$ can reduce the adverse effect of the larger position measurement error, and the estimate precisions will be improved. 3) The selection of smaller $\alpha_{3} \in(0,1)$ can improve the estimate precisions, and relatively large $\alpha_{3}$ can reject much high-frequency noise.

\section{CONCLUSION}

A NSCO has been developed. It can reject the large sensor error in position and also can estimate the unknown velocity in spite of the existence of stochastic non-Gaussian noise. The proposed scheme demonstrated by experiment, that it succeeded in rejecting the large measurement error in position, and in estimating the unknown flying velocity. The merits of the presented NSCO include its synchronous signal estimation and measurement error reduction, sufficient stochastic nonGaussian noise rejection and no drift phenomenon.

\section{APPENDIX}

Proof of Theorem 1: The error system between the NSCO (5) and the derivatives of $a_{01}(t)$ is given by:

$$
\begin{aligned}
\dot{e}_{1}= & e_{2} ; \quad \dot{e}_{2}=e_{3} ; \\
\varepsilon^{4} \dot{e}_{3}= & -k_{1}\left|\varepsilon\left(e_{1}-d_{1}(t)\right)\right|^{\alpha_{1}} \operatorname{sign}\left(e_{1}-d_{1}(t)\right) \\
& -k_{2}\left|\varepsilon^{2}\left(e_{2}+a_{02}(t)\right)\right|^{\alpha_{2}} \operatorname{sign}\left(e_{2}+a_{02}(t)\right) \\
& -k_{3}\left|e_{3}-d_{3}(t)\right|^{\alpha_{3}} \operatorname{sign}\left(e_{3}-d_{3}(t)\right) \\
& -\varepsilon^{4} \dot{a}_{03}(t)
\end{aligned}
$$

Eq. (25) can be rewritten as

$$
\begin{aligned}
\frac{d \varepsilon e_{1}}{d t / \varepsilon}= & \varepsilon^{2} e_{2} ; \frac{d \varepsilon^{2} e_{2}}{d t / \varepsilon}=\varepsilon^{3} e_{2} \\
\frac{d \varepsilon^{3} e_{3}}{d t / \varepsilon}= & -k_{1}\left|\varepsilon e_{1}-\varepsilon d_{1}(t)\right|^{\alpha_{1}} \operatorname{sign}\left(e_{1}-d_{1}(t)\right) \\
& -k_{2}\left|\varepsilon^{2} e_{2}+\varepsilon^{2} a_{02}(t)\right|^{\alpha_{2}} \operatorname{sign}\left(e_{2}+a_{02}(t)\right) \\
& -\frac{k_{3}}{\varepsilon^{3 \alpha_{3}}}\left|\varepsilon^{3} e_{3}-\varepsilon^{3} d_{3}(t)\right|^{\alpha_{3}} \operatorname{sign}\left(e_{3}-d_{3}(t)\right) \\
& -\varepsilon^{4} \dot{a}_{03}(t)
\end{aligned}
$$

Selecting the coordinate transform

$$
\begin{aligned}
& \tau=t / \varepsilon ; z_{i}(\tau)=\varepsilon^{i} e_{i} ; z=\left[\begin{array}{lll}
z_{1} & z_{2} & z_{3}
\end{array}\right]^{T} ; \\
& \bar{d}_{i}(\tau)=\varepsilon^{i} d_{i}(t), i=1,3 ; \bar{d}_{2}(\tau)=\varepsilon^{2} a_{02}(t) ; \\
& \bar{d}_{4}(\tau)=\varepsilon^{4} \dot{a}_{03}(t)
\end{aligned}
$$

we obtain $z=\Xi(\varepsilon) e$, and Eq. (26) can be written as

$$
\begin{aligned}
\frac{d z_{1}}{d \tau}= & z_{2} ; \quad \frac{d z_{2}}{d \tau}=z_{3} \\
\frac{d z_{3}}{d \tau}= & -\sum_{i=1}^{2} k_{i}\left|z_{i}\right|^{\alpha_{i}} \operatorname{sign}\left(z_{i}\right) \\
& -\frac{k_{3}}{\varepsilon^{3 \alpha_{3}}}\left|\left(z_{3}\right)\right|^{\alpha_{3}} \operatorname{sign}\left(z_{3}\right)+g(\tau, z(\tau))
\end{aligned}
$$

where

$$
\begin{aligned}
& g(\tau, z(\tau)) \\
= & -k_{1}\left\{\left|z_{1}-\bar{d}_{1}(\tau)\right|^{\alpha_{1}} \operatorname{sign}\left(z_{1}-\bar{d}_{1}(\tau)\right)\right. \\
& \left.-\left|z_{1}\right|^{\alpha_{1}} \operatorname{sign}\left(z_{1}\right)\right\} \\
& -k_{2}\left\{\left|z_{2}+\bar{d}_{2}(\tau)\right|^{\alpha_{2}} \operatorname{sign}\left(z_{2}+\bar{d}_{2}(\tau)\right)\right. \\
& \left.-\left|z_{2}\right|^{\alpha_{2}} \operatorname{sign}\left(z_{2}\right)\right\}-\bar{d}_{4}(\tau) \\
& -\frac{k_{3}}{\varepsilon^{3 \alpha_{3}}}\left\{\left|z_{3}-\bar{d}_{3}(\tau)\right|^{\alpha_{3}} \operatorname{sign}\left(z_{3}-\bar{d}_{3}(\tau)\right)\right. \\
& \left.-\left|z_{3}\right|^{\alpha_{3}} \operatorname{sign}\left(z_{3}\right)\right\}
\end{aligned}
$$

Since the nonlinear contraction mapping $\left|x^{\rho_{i}}-\bar{x}^{\rho_{i}}\right| \leq$ $2^{1-\rho_{i}}|x-\bar{x}|^{\rho_{i}}, \rho_{i} \in(0,1]$, we obtain

$$
\begin{aligned}
\delta & =\sup _{(\tau, z) \in R^{4}}|g(\tau, z(\tau))| \\
& \leq \sum_{i=1}^{2} 2^{1-\alpha_{i}} k_{i} L_{i}^{\alpha_{i}} \varepsilon^{i \alpha_{i}}+\varepsilon^{4} L_{a}+2^{1-\alpha_{3}} k_{3} L_{3}^{\alpha_{3}} \\
& \leq \varepsilon^{\rho} \delta_{0}+2^{1-\alpha_{3}} k_{3} L_{3}^{\alpha_{3}}
\end{aligned}
$$

where, $\delta_{0}=\sum_{i=1}^{2} 2^{1-\alpha_{i}} k_{i} L_{i}^{\alpha_{i}}+L_{a}$, and $\rho=$ $\min _{i \in\{1,2,3\}}\left\{\min \left\{4, i \alpha_{i}\right\}\right\}=\alpha_{1}$.

From Proposition 8.1 in [18], Theorem 5.2 in [19] and Eq. (30), for system (28), there exist positive constants $\mu$ and $\Gamma(z(0))$, such that, for $\forall \tau \in[\Gamma(z(0)), \infty)$,

$$
\|z(\tau)\| \leq \mu \delta^{\gamma} \leq \mu\left(\varepsilon^{\alpha_{1}} \delta_{0}+2^{1-\alpha_{3}} k_{3} L_{3}^{\alpha_{3}}\right)^{\gamma}
$$


where $\mu$ is a constant defined in Theorem 5.2 [19]. Therefore, from coordinate transformation (27), we obtain

$$
\left\|\varepsilon e_{1} \quad \varepsilon^{2} e_{2} \quad \varepsilon^{3} e_{3} \quad\right\| \leq \mu\left(\varepsilon^{\alpha_{1}} \delta_{0}+2^{1-\alpha_{3}} k_{3} L_{3}^{\alpha_{3}}\right)^{\gamma}
$$

for $\forall t \in[\varepsilon \Gamma(\Xi(\varepsilon) e(0)), \infty)$. Thus, the following inequality holds:

$$
\left|e_{i}\right| \leq L\left(\delta_{d i}\right)^{\gamma}, i=1,2,3, \forall t \in[\varepsilon \Gamma(\Xi(\varepsilon) e(0)), \infty)
$$

where $L=\mu \delta_{0}^{\gamma} ; \delta_{d i}=\varepsilon^{\alpha_{1}-\frac{i}{\gamma}}+\frac{2^{1-\alpha_{3}} k_{3} L_{3}^{\alpha_{3}}}{\delta_{0}} \varepsilon^{-\frac{i}{\gamma}}, i=1,2,3$. If $\varepsilon \in(0,1)$ and $L_{3}<\left(\frac{1-\varepsilon^{\alpha_{1}}}{2^{1-\alpha_{3} k_{3}}} \delta_{0}\right)^{\frac{1}{\alpha_{3}}}$, then

$$
0<\varepsilon^{\alpha_{1}}+\frac{2^{1-\alpha_{3}}}{\delta_{0}} k_{3} L_{3}^{\alpha_{3}}<1
$$

Furthermore, from Theorems 4.3 and 5.2 in [19], $\gamma$ can be chosen to be arbitrarily large. Hence, the requirement that $\gamma$ lies on

$$
\gamma>\max \left\{\frac{4 \log \varepsilon}{\log \left(\varepsilon^{\alpha_{1}}+\frac{2^{1-\alpha_{3}}}{\delta_{0}} k_{3} L_{3}^{\alpha_{3}}\right)}, 1\right\}
$$

is not restrictive. Therefore,

$$
\gamma \log \left(\varepsilon^{\alpha_{1}}+\frac{2^{1-\alpha_{3}}}{\delta_{0}} k_{3} L_{3}^{\alpha_{3}}\right)<4 \log \varepsilon
$$

i.e.,

$$
\varepsilon^{\alpha_{1}}+\frac{2^{1-\alpha_{3}}}{\delta_{0}} k_{3} L_{3}^{\alpha_{3}}<\varepsilon^{\frac{4}{\gamma}}
$$

From Eq. (35), $\gamma>4$ holds. Therefore, from $\varepsilon \in(0,1)$, we can obtain $\varepsilon^{\frac{4}{\gamma}}<\varepsilon^{\frac{i}{\gamma}}, i=1,2,3$. Then

$$
\delta_{d i}=\varepsilon^{\alpha_{1}-\frac{i}{\gamma}}+\frac{2^{1-\alpha_{3}}}{\delta_{0}} k_{3} L_{3}^{\alpha_{3}} \varepsilon^{-\frac{i}{\gamma}}<1
$$

where $i=1,2,3$. The choice of $\gamma$ leads to $\gamma>1$ in (33) which implies that for $\delta_{d i} \in(0,1)$, the ultimate bound (33) on the estimation error is of higher order than the perturbation. Consequently, the NSCO leads to perform rejection of persistent disturbances.

Furthermore, assume there is no sensor error in signal $a_{3}(t)$, i.e, $a_{3}(t)=a_{03}(t)$ or $L_{3}=0$, then (33) can be written as

$$
\left|e_{i}\right| \leq L \varepsilon^{\alpha_{1} \gamma-i}, i=1,2,3, \forall t \in[\varepsilon \Gamma(\Xi(\varepsilon) e(0)), \infty)
$$

We know that $\alpha_{1} \gamma-i>1, i=1,2,3$. In fact, from Theorems 4.3 and 5.2 in [19], $\gamma$ can be chosen to be arbitrarily large, and

$$
\gamma>\max \left\{\frac{4}{\alpha_{1}}, 1\right\}=\frac{4}{\alpha_{1}}
$$

is not restrictive. Accordingly, for $i=1,2,3$, we can obtain

$$
\alpha_{1} \gamma-i>1
$$

It implies that, for $\varepsilon \in(0,1)$, the ultimate bound (39) on the estimation error is of higher order than the perturbation.
For arbitrary $\varepsilon \in(0,1)$, from the Routh-Hurwitz Stability Criterion, $s^{3}+\frac{k_{3}}{\varepsilon^{3 \alpha_{3}}} s^{2}+k_{2} s+k_{1}$ is Hurwitz if $k_{1}>0, k_{3}>0$, $k_{2}>\varepsilon^{3 \alpha_{3}} k_{1} / k_{3}$. This concludes the proof.

\section{REFERENCES}

[1] D. Odijk, N. Nadarajah, S. Zaminpardaz, P.J. G. Teunissen. GPS, Galileo, QZSS and IRNSS differential ISBs: estimation and application, GPS Solutions, vol. 21, no. 2, 439-450, Apr. 2017.

[2] M.F. Abdel-Hafez. Detection of bias in GPS satellites' measurements: A probability ratio test formulation, IEEE Trans. Control Syst. Technol., Vol. 22, No. 3, 1166-1173, 2014.

[3] M.S. Golsorkhi, D.D.C. Lu, J.M. Guerrero. A GPS-Based Decentralized Control Method for Islanded Microgrids, IEEE Transactions on Power Electronics, vol. 32, no. 2, 1615-1625, Feb. 2017.

[4] L.R.G. Carrillo, I. Fantoni, and E. Rondon. Three-dimensional position and velocity regulation of a quad-rotorcraft using optical flow, IEEE Trans. Aerosp. Electron. Syst., vol. 51, no. 1 , 358-371, 2015.

[5] M. Lungu R. Lungu. Design of full-order observers for Systems with unknown inputs by using the eigenstructure assignment. Asian J. Control, vol. 16, no. 5, pp. 1470-1481, 2014.

[6] Z. Pu, R. Yuan, J. Yi, X. Tan. A class of adaptive extended state observers for nonlinear disturbed systems, IEEE Trans. Ind. Electron., vol. 62, no. 9, 5858-5869, Sept. 2015.

[7] M. Lungu, R. Lungu. Reduced-order multiple observer for aircraft state estimation during landing. Applied Mechanics and Materials, vol. 841, pp. 253-259, 2016.

[8] M.K. Jalloul, M.A. Al-Alaoui. Design of recursive digital integrators and differentiators using particle swarm optimization, Int. J. Circ. Theor. App., vol. 44, no. 5, pp. 948-967, 2016.

[9] A. Aggarwal, T.K. Rawat, D.K. Upadhyay. Optimal design of L1-norm based IIR digital differentiators and integrators using the bat algorithm, IET Signal Processing, vol. 11, no. 1, pp. 26-35, Feb. 2017.

[10] J.M. Hansen , T.A. Johansen, N. Sokolova, T.I. Fossen. Nonlinear Observer for Tightly Coupled Integrated Inertial Navigation Aided by RTK-GNSS Measurements, IEEE Trans. Control Syst. Technol., DOI: 10.1109/TCST.2017.2785840

[11] X. Wang, B. Shirinzadeh, and M.H. Ang,Jr. Nonlinear doubleintegral observer and application to quadrotor aircraft, IEEE Trans. Ind. Electron., vol. 62, no. 2, 1189-1200, 2015.

[12] R.H. Rogne, T.H. Bryne, T.I. Fossen, T.A. Johansen. Redundant MEMS-based inertial navigation using nonlinear Observers, $J$. Dyn. Sys., Meas., Control, vol. 140, no. 7, 071001 1-7, Jul. 2018.

[13] D.A. Mercado, G. Flores, P. Castillo1, J. Escareno, and R. Lozano1. GPS/INS/Optic flow data fusion for position and velocity estimation, 2013 International Conference on Unmanned Aircraft Systems (ICUAS), Grand Hyatt Atlanta, Atlanta, GA, May 28-31, 2013, 486-491.

[14] Y. Kim, J. An, J. Lee. Robust navigational system for a transporter using GPS/INS fusion, IEEE Trans. Ind. Electron., vol. 65, no. 4, 3346-3354, Apr. 2018.

[15] M.A.K. Jaradat, M.F. Abdel-Hafez. Non-linear autoregressive delay-dependent INS/GPS navigation system using neural networks, IEEE Sensors J., vol. 17, no. 4, pp. 1105-1115, Feb. 2017.

[16] F. Auger, M. Hilairet, J.M. Guerrero, E. Monmasson. Industrial applications of the Kalman filter: A Review, IEEE Trans. Ind. Electron., vol. 60, no. 12, 5458-5471, Dec. 2013.

[17] L. Idkhajine, E. Monmasson, A. Maalouf. Fully FPGA-based sensorless control for synchronous AC drive Using an Extended Kalman filter, IEEE Trans. Ind. Electron., vol. 59, no. 10, 39083918, Oct. 2012.

[18] S.P. Bhat and D.S. Bernstein. Geometric homogeneity with applications to finite-time stability, Math. Control, Signals, Syst., vol. 17, no. 2, 101-127, Jun. 2005.

[19] S.P. Bhat and D.S. Bernstein. Finite-time stability of continuous autonomous systems, Siam J. Control Optim., vol. 38, no. 3, 751-766, Mar. 2000.

[20] M.A. Khosravi, H.D. Taghirad. Dynamic modeling and control of parallel robots with elastic cables: singular perturbation approach, IEEE Trans. Robot., vol. 30, no. 3, pp. 694-704, Jun. 2014. 
[21] A. Tayebi and S. McGilvray, Attitude stabilization of a VTOL quadrotor aircraft," IEEE Trans. Control Syst. Technol., vol. 14, no. 3, pp. 562-571, May 2006.

[22] S. Islam, P.X. Liu, A.E. Saddik. Observer-based adaptive output feedback control for miniature aerial vehicle, IEEE Trans. Ind. Electron., vol. 65, no. 1, 470-477, Jan. 2018.

[23] F. Chen, R. Jiang, K. Zhang, B. Jiang, G. Tao. Robust backstepping sliding-mode control and observer-based fault estimation for a quadrotor UAV, IEEE Trans. Ind. Electron., vol. 63, no. 8, 5044-5056, Aug. 2016.

[24] M. Lungu, R. Lungu, C. Rotaru. New systems for identification, estimation and adaptive control of the aircrafts movement. Studies in Informatics and Control, vol. 20, no. 3, pp. 273-284, 2011.

[25] K. Sebesta and N. Boizot. A real-time adaptive high-gain EKF, applied to a quadcopter inertial navigation system, IEEE Trans. Ind. Electron., vol. 61, no. 1, pp. 495-503, Jan. 2014.

[26] T. Hamela and R. Mahony. Image based visual servo control for a class of aerial robotic system, Automatica, vol. 43, no. 11, pp. 1976-1983, Nov. 2007

[27] N. Guenard, T. Hamel, and R. Mahony. A practical visual servo control for an unmanned aerial vehicle, IEEE Trans. Robot., vol. 24, no. 2, pp. 331-340, Apr. 2008.

[28] A. Levant. High-order sliding modes, differentiation and outputfeedback control, Int. J. of Control, vol. 76, no. 9/10, 924-941, 2003.

[29] R. Baltes, A. Schultschik, O. Farle, and R. Dyczij-Edlinger. A finite-element-based fast frequency sweep framework including excitation by frequency-dependent waveguide mode patterns, IEEE Trans. Microw. Theory Techn., vol. 65, no. 7, pp. 22492260, Jul. 2017.

[30] X. Wang and H. Lin. Design and frequency analysis of continuous finite-time-convergent differentiator, Aerosp. Sci. Technol, vol. 18, no. 1, pp. 69-78, Apr.-May 2012. 